\title{
Travel with hydrogen
}

\section{In the field of transportation, hydrogen does not have a particularly glorious history. Just think of the dozens of hydrogen airships destroyed by fire over the years, with the Hindenburg disaster in 1937 as the most famous example. Now $\mathrm{H}_{2}$ is trying a comeback on the road, often in combination with a fuel cell and an electric motor to power the car.}

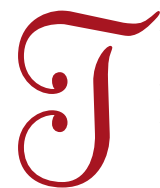
he problem, of course, is that finding a compact and light-weight storage medium for hydrogen is not easy. The reason is the very nature of the $\mathrm{H}_{2}$ molecule. Consisting of only two protons and two electrons, the intermolecular attractive forces are weak, which in turn makes the attractive potential well very shallow. The first consequence is that the boiling point is quite low: $20.4 \mathrm{~K}$. Storing $\mathrm{H}_{2}$ in liquid form therefore requires a cryogenic container. The second consequence is that it takes only little energy for the molecules to escape from the potential well: the heat of vaporization is quite low. So if you want to store liquid hydrogen in your car, the 'boil-off' is a serious problem; you don't want to return from a conference and find your fuel tank empty.

So, for application in automobiles a high-pressure tank seems to be the better option. But also here we face a slight drawback. Since the repulsive forces are far dominant at ambient temperatures, compression of the gas does not follow the ideal-gas law. For example, at 700 bar - which

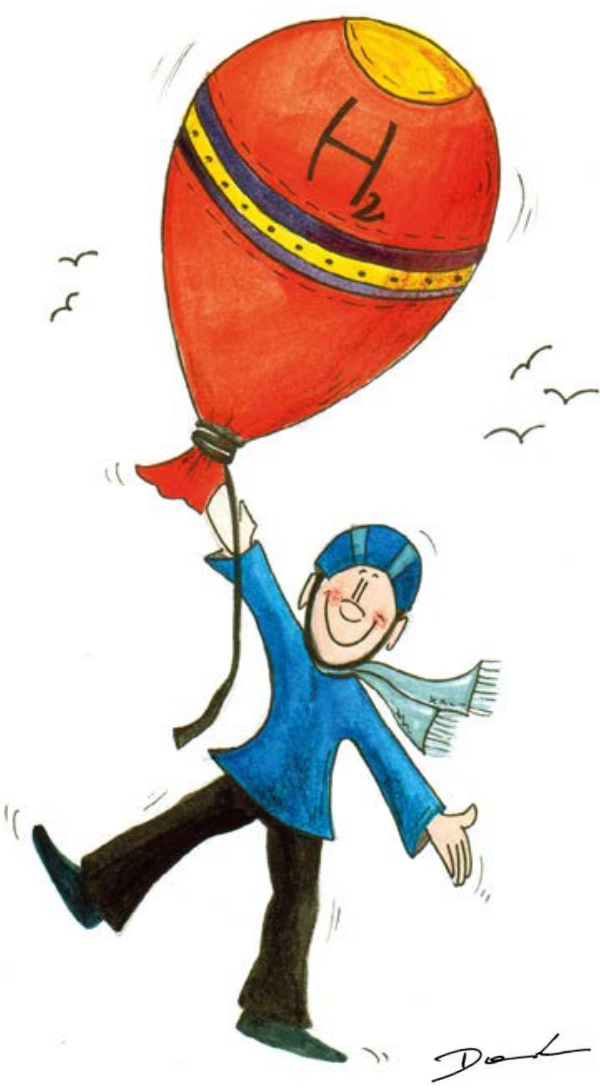
is the pressure in use nowadays - the density is only $2 / 3$ of what one would expect naively.

How does this compare with storing energy in batteries? In contrast to $\mathrm{H}_{2}$, where progress is frustrated by the nature of the molecule, in the case of batteries progress is determined by technology. And indeed, tremendous progress has been made, especially for Li-ion batteries.
The gravimetric energy density has improved by a factor of 3 since their launch in 1991 and has now reached 0.25 $\mathrm{kWh} / \mathrm{kg}$, while cost has fallen by a factor of ten over the last two decades. So one is tempted to conclude that the battle is over: batteries have won, and we can forget about hydrogen for transportation.

But wait! Couldn't hydrogen play a role in aviation, where weight is such a crucial factor? After all, the gravimetric energy density of hydrogen is exceptionally high: three times the value for kerosene. This is especially important for long-haul flights, where the weight of the kerosene is roughly equal to the weight of the empty aircraft. Let us take a bold step and think of liquid hydrogen as a future fuel for aircraft. Most of the drawbacks which one faces when using liquid hydrogen in cars are no longer valid for use in aviation. For one thing, boil-off is much less of a problem: aircraft are airborne only for a limited amount of time, and moreover the low outside temperature at cruising altitude reduces the temperature difference with the liquid. In addition, refueling the cryogenic liquid, which may pose risks if done by laymen filling up the tank of their car, will be exclusively done by professionals at airports, thus reducing safety issues to the level of handling kerosene.

So, who knows, our grandchildren may take to the skies with hydrogen, not using the gas to lift an airship - slow like a snail - but to fuel the engines of a hydrogen airliner - at supersonic speed. 